\title{
Functional genomics of autoimmune diseases
}

\author{
Akari Suzuki, Matteo Maurizio Guerrini, Kazuhiko Yamamoto
}

Handling editor Josef S Smolen

Laboratory for Autoimmune Diseases, RIKEN Center for Integrative Medical Sciences, Yokohama, Kanagawa, Japan

\section{Correspondence to}

Dr Kazuhiko Yamamoto, Laboratory for Autoimmune Diseases, RIKEN Center for Integrative Medical Sciences, Yokohama 230-0045,

Kanagawa, Japan;

kazuhiko.yamamoto@riken.jp

Received 30 October 2020 Accepted 6 December 2020 Published Online First 6 January 2021

Check for updates

(C) Author(s) (or their employer(s)) 2021. No commercial re-use. See rights and permissions. Published by BMJ.

To cite: Suzuki A, Guerrini MM, Yamamoto K. Ann Rheum Dis

2021;80:689-697.

\section{ABSTRACT}

For more than a decade, genome-wide association studies have been applied to autoimmune diseases and have expanded our understanding on the pathogeneses. Genetic risk factors associated with diseases and traits are essentially causative. However, elucidation of the biological mechanism of disease from genetic factors is challenging. In fact, it is difficult to identify the causal variant among multiple variants located on the same haplotype or linkage disequilibrium block and thus the responsible biological genes remain elusive. Recently, multiple studies have revealed that the majority of risk variants locate in the non-coding region of the genome and they are the most likely to regulate gene expression such as quantitative trait loci. Enhancer, promoter and long non-coding RNA appear to be the main target mechanisms of the risk variants. In this review, we discuss functional genetics to challenge these puzzles.

\section{INTRODUCTION}

Autoimmune diseases, such as rheumatoid arthritis, are thought to arise from the interaction of genetic and environmental factors. The contribution of genetic factors to autoimmune diseases has been examined for more than 40 years. Initial investigations focused on major histocompatibility complex genes and were followed by those that focused on several other candidate genes. Improvements in commercial arrays since 2007 has led to the development of the current form of genome-wide association studies (GWASs). GWASs have been applied in many studies investigating autoimmune diseases and have expanded our understanding of their underlying genetic factors. GWASs rely on single-nucleotide polymorphisms (SNPs) and haplotype blocks, which are distributed across all chromosomes. A number of representative tag SNPs-500000 to several millions-are selected based on the haplotype information and combined into a single array for typing. The loci associated with disease susceptibility can then be determined by comparing variants represented by tag SNPs between patients and controls and identifying those that are significantly different.

GWASs have helped identify thousands of loci as being statistically associated with a risk for various diseases and traits. ${ }^{1}$ The same loci have also been identified in other independent analyses of the same diseases, suggesting that the associations determined using GWASs hold true. ${ }^{2}$ Genetic risk factors are essentially causative for specific diseases and traits; however, understanding the biological mechanism of risk from a genetic factor is challenging. Although there are only three possible patterns, with corresponding intermediate phenotypes, in which risk variants are involved in the development of a disease (figure 1), the types of change brought about by risk variants are complex. Furthermore, many variants significantly associated with each other are often in strong linkage disequilibrium (LD) and form a haplotype. ${ }^{3}$ Thus, although associations between variants and diseases can be identified, it is difficult to identify the causal variant among multiple variants located on the same haplotype ${ }^{4}$ and experimental validations are needed to determine the functional variants. ${ }^{5}$ Furthermore, understanding the biological functions of risk variants is challenging, since more than $80 \%$ of diseaseassociated variants are located in non-coding regions of the genome. ${ }^{6}$ Transcriptome analysis can offer some clues regarding how these variants alter gene regulation and the expression of splice variants of target genes, ${ }^{7}$ but the detailed relationship between risk variants and genes is largely unknown.

Analyses that integrate various 'omics' studies and GWAS data have emerged as powerful tools for understanding the functions of risk variants identified in a GWAS. The capabilities of next-generation sequencing techniques for analysing the functions of non-coding regions have advanced dramatically in recent years, enabling the comprehensive analysis of enhancers, promoters, histone modifications and chromatin structures. Analysis of the expression of quantitative trait loci (QTL) is used to investigate how particular variants lead to intermediate phenotypes (eg, epigenetic statuses). QTL are DNA markers on a chromosome that indicate genes involved in a quantitative trait. ${ }^{8}$ Recently, QTL analysis has been used to identify loci that are associated with a particular quantitative phenotypic trait or disease and can be caused to polygenic effects. ${ }^{9}$ Therefore, QTL analysis has been used for assessing human polygenic diseases and phenotypes as well as intermediate phenotypes, such as gene expression.

In this review, we discuss the possible functional genomic strategies for integrating GWAS results with the current understanding of specific diseases. In particular, we focus on promoters, enhancers and long non-coding RNAs. We also list a suite of analytical methods for the functional dissection of disease-associated risk loci. We believe that functional genomics will enhance our understanding of the immunological and biological functions of genes.

\section{FUNCTIONS OF GWAS-IDENTIFIED RISK VARIANTS}

The ever-increasing number of genome-wide genotyping arrays has enabled the imputation of variants and the compilation of larger and more accurate reference datasets. ${ }^{10-12}$ Identifying the function of risk variants is important for understanding the mechanism of disease onset. Indeed, several risk variants affect the structures of proteins, for example, by inducing amino acid alterations or 


\section{Causal}

Genotype acts on the disease directly or through the intermediate phenotype
Consequential

The intermediate phenotype is the consequence of the diseases


Independent

Genotype acts on the intermediate phenotype and diseases independently

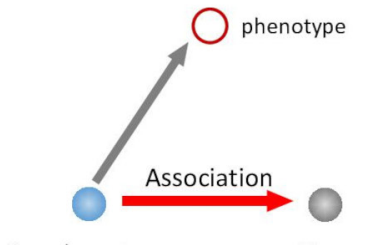

Figure 1 Risk patterns of causative variants and intermediate phenotypes. Disease development involves a variety of characteristic intracellular and internal events. These are collectively referred to as intermediate phenotypes when we consider a causal genotype and the disease outcome. Intermediate phenotypes involve gene expression, protein expression and epigenetic effects and others. There are three possible patterns via which causative variants are associated with disease. First, a risk-associated genotype acts on the disease via the intermediate phenotype, which is assigned as the causal intermediate phenotype. Second, the intermediate phenotype is not causal but the consequence of the disease. Third, intermediate phenotypes are independent from the disease.

splicing variations. However, the functions of the majority (more than $80 \%$ ) of risk variants are unknown because many of them are located in non-coding genomic regions. To elucidate the functions of risk variants, more detailed GWAS and omics data regarding intermediate phenotypes of the whole genome are necessary.

Post-GWAS omics analyses can improve our understanding of the molecular events orchestrating the development of diseases and traits. In particular, high-throughput transcriptomic ${ }^{37}$ and genome-wide epigenomic ${ }^{3}$ analyses are commonly used omics analyses that can be exploited to identify QTL. QTL identified through omics analyses overlapping with GWAS-identified risk variants hint at the pathogenetic mechanism of a disease. There are various types of QTL, which we have described below. The most common QTL type is expression QTL (eQTL), which is defined as a variant directly related to the expression level.

\section{Expression QTL}

The majority of disease-associated variants reside in non-coding regions of the genome, suggesting that they regulate gene expression. Indeed, a combination of statistical analysis, imputed genotypes in LD, transcriptomics and epigenomics has helped identify a number of eQTL that overlap with variants implicated in autoimmune diseases. ${ }^{3}$ Since eQTL are strongly influenced by epigenetic changes, they are often observed in conjunction with epigenetic QTL (figure 2). In many cases, eQTL function as cis-eQTL, which directly affect the expression of nearby genes (usually within $250 \mathrm{~kb}$ to $1 \mathrm{Mb}$ ) in an allele-specific manner. ${ }^{713} 14$ In contrast, eQTL that are located at distance from their gene-oforigin, often on different chromosomes, are referred to as transeQTL. Some cis-eQTL associated to house-keeping genes, are observed in many different tissue types; however, the majority of eQTL are highly tissue specific. ${ }^{12}$ The $3 \mathrm{D}$ map of chromatin interaction can be used to contextualise eQTLs. Hi-C and other chromosome conformation capture technologies provide a quantitative and qualitative description of the $3 \mathrm{D}$ arrangement of the genome and further our understanding of gene regulation. ${ }^{15}$

\section{Splicing QTL}

Splicing QTL (sQTL), a subtype of eQTL, enable the quantitation of genetic variation due to the expression of RNA isoforms from alternative splicing events. sQTL are thought to contribute significantly to disease development because splicing is a major post-transcriptional modification and the primary mechanism governing protein diversity. Alternative splicing affects multiexonic genes, often in a cell type-specific fashion, and modulates phenotypic changes in a flexible and dynamic manner. ${ }^{16}$ RNA-sequencing (RNA-seq) is used for analysing both eQTL and sQTL. ${ }^{16}$ In multiple sclerosis, the estimated effect size of sQTL is larger than that of eQTL, supporting the well-known importance of RNA splicing in the brain. ${ }^{3}$ Thus, RNA splicing is a promising area of investigation to understand the effect of genetic variation in a complex disease.

\section{Epigenetic QTL, including DNA methylation QTL and histone modification QTL}

Epigenetic events are classically defined as post-translational modifications that determine chromatin status, including DNA methylation and multiple types of histone modifications (eg, $\mathrm{H} 3 \mathrm{~K} 4 \mathrm{me} 1, \mathrm{H} 3 \mathrm{~K} 4 \mathrm{me} 3$ and $\mathrm{H} 3 \mathrm{~K} 27 \mathrm{ac}$; figure 2). DNA methylation QTL (meQTL) and histone modification QTL are directly affected by their different genotypes. Epigenetic QTL are cell type and context specific. ${ }^{17}$ Indeed, epigenetic QTLs are highly specific for disease conditions, such as malignancy, or the effects of specific drugs. It is tempting to speculate that the high degree of specificity inherent to epigenetic QTL can be exploited to identify novel patient-specific therapeutic targets.

\section{Assay for transposase-accessible chromatin QTL and DNase I hypersensitive site QTL}

Gene expression and epigenetic events involve chromatin status, which is affected by both genetic variants and environmental factors. DNase I hypersensitive sites (DHS) indicate an open chromatin structure influence and are influenced by transcriptional regulatory elements and histone modifications (figure 2)..$^{9}$ Assay for transposase-accessible chromatin sequencing (ATAC-seq) also detects open chromatin. Genetic variants that are correlated with the phenotypes of DHS and ATAC are called DHS-QTL and ATAC-QTL, respectively. These QTL contribute to differences in gene regulation between individuals. ${ }^{18}$

\section{Transcription factor-binding site QTL}

Sequence-specific transcription factors (TFs) regulate gene expression by binding to cis-regulatory elements in the promoter and enhancer regions. TFs typically recognise binding-site motifs with particular DNA sequences (figure 2). Variants in DNA TF binding sites are designated as TF-binding site QTL (bQTL). For 

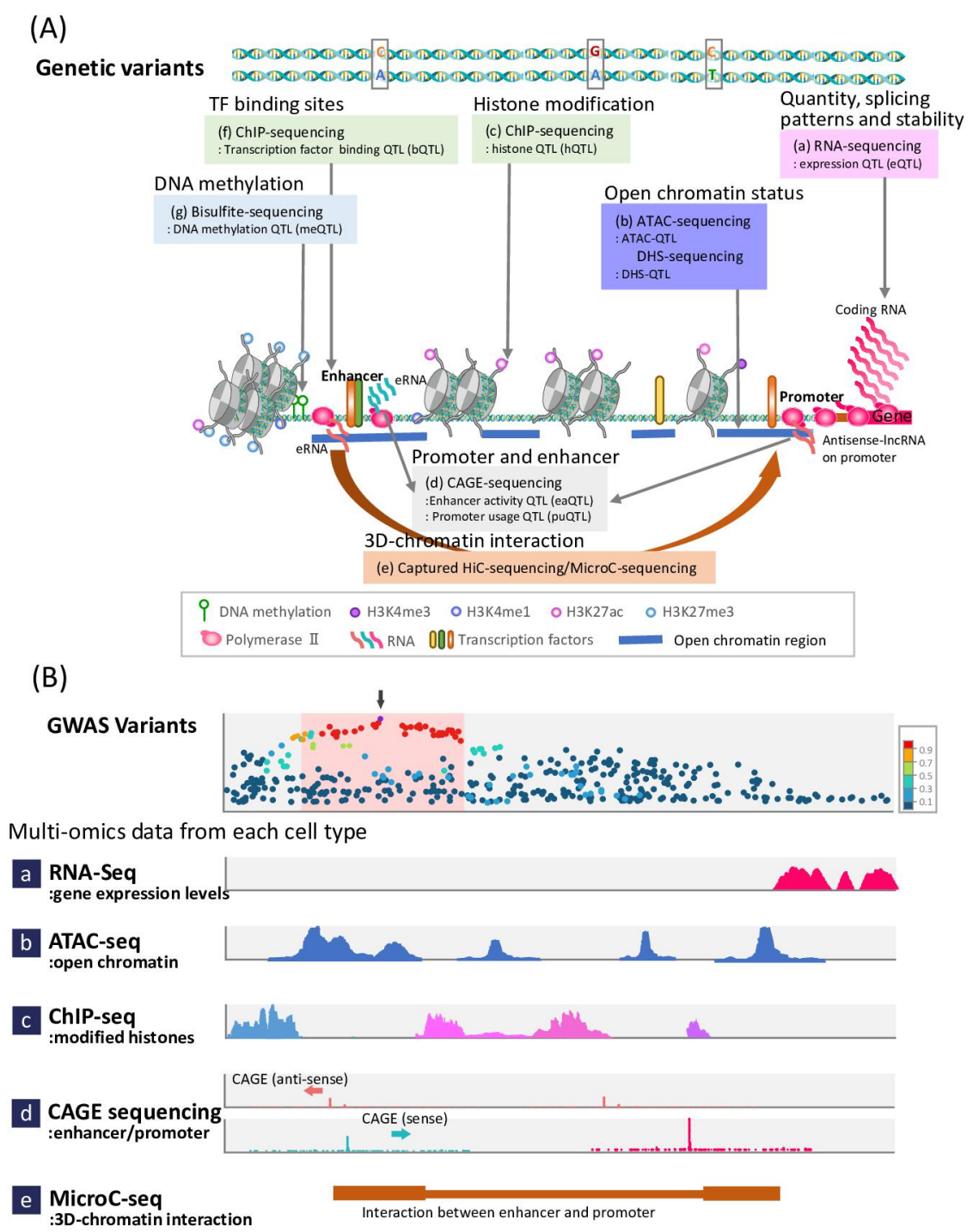

Figure 2 Genetic variants and quantitative trait loci (QTL). (A) Genetic variants can affect different types of transcriptional and post-transcriptional regulatory factors, including (a) gene expression level, (b) open chromatin status, (c) histone modifications, (d) promoter usage and enhancer activity, (e) chromatin conformation, (f) transcription factor binding sites, and (g) DNA methylation. (B) Actually, genome-wide association studies (GWASs) results integrate a variety of omics layer data constructed using the latest technologies (eg, the eQTL study involves RNA-seq for exploring gene expression profiles). In the upper row, the regional association plot of GWAS is presented. The purple dot indicates the top single-nucleotide polymorphism (SNP) (SNP with the smallest $p$ value). SNPs highly correlated with the top SNP are equally likely to be causal in the LD block (pink box), demonstrating how omics data can be combined with GWAS findings to provide insights into causal variants and genes. These SNPs are usually outside the gene body (pink peaks, a) and are located in the regulatory region of the gene. The region is open (assay for transposaseaccessible chromatin (ATAC)-peaks (blue), b) and flanked by modified histones (light blue and pink peaks, c). The non-coding RNA-expressed region (d) indicates active promoter and enhancer positions, which interact (Micro-C, brown bars, e) with each other. Integration of these data for each cell provides evidence for genes and cell types implicated in the disease GWAS findings. CAGE, cap analysis of gene expression; ChIP, chromatin immunoprecipitation.

example, the binding site of transcription factor 21 has been reported to have bQTL effects. ${ }^{19}$

\section{Promoter usage QTL and enhancer activity QTL}

eQTL variants are typically enriched in regulatory regions. In particular, variants located in promoter regions are associated with alternative transcript usage and include variations in the mRNA 5'-end. These are termed promoter usage QTL (puQTL; figure 2). ${ }^{18}$ On average, there are more than four promoters per human gene, indicating that studies of puQTL are important for understanding eQTL effects. ${ }^{18}$

Enhancers are located remotely from promoters, and, unlike promoters, they regulate gene expression in a distance-independent manner by impacting transcriptional timing and cell-specificity of gene expression. Variants related to enhancer activity are referred to as enhancer activity QTL (eaQTL; figure 2). Methods that capture the 5'-end of a transcript are often used to identify puQTL and eaQTL. ${ }^{20}$

\section{Non-coding RNA QTL}

Micro RNAs (miRNAs) and long non-coding RNAs (lncRNAs) represent a class of non-coding RNAs that act as posttranscriptional regulators of gene expression and mRNA translation. $^{21}$ miRNAs and lncRNAs are transcripts composed of $21-25 \mathrm{bp}$ and $>200 \mathrm{bp}$, respectively, and are extensively reported to be involved in transcriptional regulation (figure 1). 
We describe the relationship between promoters, enhancers and long non-coding RNAs in detail below.

\section{PROMOTERS AND RISK VARIANTS}

Promoters are regulatory modules located upstream of the transcription start site (TSS). They are approximately 100-1000 bp long and contain a core region and one or more proximal regions. $^{22}$ The core promoter supports the assembly of the transcription preinitiation complex, including general TFs and RNA polymerase (Pol) II. ${ }^{23}$ Core promoters control the magnitude of transcription, but not its induction, by regulating the binding of RNA Pol II at the TSS. Once the RNA Pol II reaches a few bases downstream of the TSS, further transcript elongation requires the binding of TFs and cofactors to the proximal region of the promoter. $^{24-26}$

Genetic variants located in promoter regions, originally designated as eQTL, influence the strength and/or stability of the interaction between TFs and the promoter of an immune systemrelated gene, likely modifying its expression pattern and thus facilitating the onset of autoimmune diseases. ${ }^{27}$ For example, a variant altering the binding affinity for the transcription factor $\mathrm{C} / \mathrm{EBP} \beta$ in the promoter of FasL increases the risk of developing systemic lupus erythematosus. ${ }^{28}$ Similarly, an eQTL variant in the promoter of FCRL3, highly expressed in B cells, increases its affinity for NF- $\mathrm{BB}$ binding, rendering it a risk factor for rheumatoid arthritis. ${ }^{29}$

Promoters are widespread; thus, promoter risk variants that alter gene expression cannot always be determined by eQTL analysis but are revealed by puQTL analysis. Cap analysis of gene expression (CAGE), which detects the 5'-end of target transcripts, can help detect puQTL and eaQTL. ${ }^{20} 30$ In some cases, the results of CAGE are not linked to eQTL results. For example, TTC23 has two promoter regions $1.6 \mathrm{~kb}$ apart; rs8028374 was mapped as a puQTL with significant opposite effects on the two promoter regions. However, TTC23 has not been identified as an eQTL. ${ }^{18}$

Risk variants are found to be enriched in regulatory elements and are therefore thought to regulate gene expression levels, often in a cell type- and context-specific manner (eg, stimulation and disease conditions). Overall, the relative participation of alternative promoters in the transcriptional output of genes, a previously underappreciated factor affecting disease development and complex trait associations, but the integration of puQTL and eQTL in various cell types and cell conditions provides new insights into the mechanisms underlying eQTL and helps explain the prevalence of specific transcript isoforms. ${ }^{18}$

\section{ENHANCERS AND RISK VARIANTS}

Enhancers are distal regulatory modules that boost the transcription of associated promoters, irrespective of their orientation. The first enhancer was identified in vitro decades ago as a distal sequence that increased the transcription of a reporter gene under the control of the SV40 promoter. ${ }^{24}$ Enhancers typically span $50-1500 \mathrm{bp}$ and contain multiple TF binding sites, enabling them to orchestrate proper spatiotemporal gene expression during development ${ }^{31}$ or in response to signalling molecules. ${ }^{32}$ Enhancers are structurally and functionally similar to the proximal regions of promoters. Indeed, both promoters and enhancers are bound by multiple TFs, support the formation of the preinitiation complex, and recruit RNA Pol II. ${ }^{33}$ Functionally, both promoters and enhancers support bidirectional transcription and activate transcript elongation. ${ }^{33}$ In promoters, the sense strand expresses mRNA and the antisense strand expresses promoter upstream transcripts (PROMPT/uaRNA). In enhancers, both the sense strand and the antisense strand express non-coding enhancer RNAs (eRNA) which are short, typically unspliced and non-adenylated transcripts.

Most GWAS variants have been found to overlap with cell type-specific enhancers that have been newly annotated through epigenomic profiling (table 1). ${ }^{34}$ Enhancer variants probably play an important role in fine-tuning the transcriptional output of each cell type and therefore affect susceptibility to common diseases. A number of classical methods to identify enhancers rely on the evolutionary conservation of non-coding sequences. ${ }^{35} 36$ In a landmark study, the candidate causal variants for 21 autoimmune diseases were integrated with RNA-seq data and chromatin features of control and stimulated human $\mathrm{CD} 4{ }^{+} \mathrm{T}$-cell subsets, regulatory $\mathrm{T}$ cells, $\mathrm{CD} 8^{+} \mathrm{T}$ cells, B cells and monocytes. ${ }^{3}$ Importantly, $90 \%$ of the causal variants were located in the non-coding regions of the genome. Approximately $60 \%$ of the variants were mapped to enhancers specific to immune cells, which showed increased histone acetylation and transcriptional activity on immune stimulation. ${ }^{3}$ The same study demonstrated that causal disease variants had a high probability of being context-specific immune enhancers and suggested that most non-coding causal variants alter atypical regulatory motifs rather than recognisable consensus motifs. ${ }^{3}$ Validation of enhancers and promoters identified through GWASs has historically been highly challenging.

More recently, next-generation sequencing technologies used in large-scale epigenomics projects have enabled the mapping and characterisation of regulatory regions in the human genome. Several methods have been developed to efficiently detect enhancers, such as chromatin immunoprecipitation-sequencing (ChIP-seq), which is used to identify TF binding or a histone modification in the enhancer sequence, and the DHS-seq and ATAC-seq methods, which are used to identify the open chromatin structure of activated enhancers. These methods were initially used in combination to detect enhancers. ${ }^{37}$ As direct detection methods of regulatory regions, self-transcribing active regulatory region sequencing (STARR-seq) and CAGEsequencing have been developed, which are used for assaying the enhancer activity of millions of enhancer candidates from arbitrary sources of DNA. ${ }^{38}$ In combination with next-generation sequencing, CAGE-sequencing enables the genome-wide determination of coding and non-coding TSSs. ${ }^{20} 3339$ The resulting database is instrumental to the identification of promoters and enhancers, both of which are characterised by the bidirectional transcripts. Enhancer activation leads to the bidirectional production of low levels of eRNAs. ${ }^{40}$

\section{EFFECTS OF LNCRNAS AND OTHER TRANSCRIPTS FROM NON-CODING REGIONS}

The cumulative expression of non-coding genes is approximately four times greater than the expression of all mRNAs, making lncRNAs one of the most abundant RNA species within the average cell. ${ }^{9}$ The genomic and epigenomic characterisation of $\operatorname{lncRNA-coding}$ regions revealed that $\operatorname{lncRNAs}$ preferentially originate from enhancers rather than promoters. ${ }^{20}$ lncRNAs do not encode proteins; however, lncRNAs longer than 200 nucleotides fold into complex structures and have biological functions. Functional lncRNAs can specifically interact with proteins, DNA and other RNAs to exert their molecular functions (figure 3). Folded lncRNAs have a variety of functions, such as transcriptional regulation (figure $3 \mathrm{~A}$ ), recruitment of TFs and proteins (figure $3 \mathrm{~B}$ ), degradation of transcript and splicing variants (figure 3C), and chromatin remodelling (figure 3D). ${ }^{204142}$ 
Table 1 Genome-wide association studies (GWASs)-associated expression quantitative trait loci (eQTL) affecting gene regulatory elements

\begin{tabular}{|c|c|c|c|c|c|c|c|c|c|}
\hline \# & $\begin{array}{l}\text { GWAS-associated } \\
\text { QTL variant }\end{array}$ & Chr & $\begin{array}{l}\text { Variant genomic } \\
\text { position }\end{array}$ & $\begin{array}{l}\text { Associated } \\
\text { disease }\end{array}$ & $\begin{array}{l}\text { Affected DNA } \\
\text { regulatory element }\end{array}$ & Affected gene(s) & $\begin{array}{l}\text { Gene TSS genomic } \\
\text { position (Refseq) }\end{array}$ & $\begin{array}{l}\text { Genomic distance } \\
\text { variant to TSS (bp) }\end{array}$ & $\begin{array}{l}\text { Reference } \\
\text { paper }\end{array}$ \\
\hline 1 & rs34481144 & 11 & 320836 & Influenza infection & Core promoter & IFITM3 & 320813 & 23 & 65 \\
\hline 2 & rs71542466 & 6 & 32666728 & l & Proximal promoter & HLA-DQB1 & 32666607 & 121 & 66 \\
\hline \multirow[t]{2}{*}{3} & rs115662534(T) & 20 & 46117814 & 1 & Proximal enhancer & CD40 & 46118344 & -530 & 67 \\
\hline & rs548231435(C) & 20 & 46118145 & I & Proximal promoter & CD40 & 46118344 & -199 & \\
\hline 4 & rs4794067 & 17 & 47731462 & $\begin{array}{l}\text { Autoimmune } \\
\text { hepatitis-related to } \\
\text { SLE and periodontitis }\end{array}$ & Proximal enhancer (?) & TBX21 (Tbet) & 47733455 & -1993 & 68 \\
\hline 5 & rs10750836 & 11 & 69048055 & SLE & $\begin{array}{l}\text { Proximal promoter/ } \\
\text { proximal enhancer }\end{array}$ & TPCN2 & 69048998 & -943 & 69 \\
\hline 6 & rs2275675 & 1 & 183470348 & SLE & $\begin{array}{l}\text { Proximal promoter/ } \\
\text { proximal enhancer }\end{array}$ & SMG7 & 183472621 & -2273 & 70 \\
\hline 7 & rs6426749 & 1 & 22384980 & Osteoporosis & Enhancer & LINC00339 & 22025504 & 359476 & 71 \\
\hline \multirow[t]{2}{*}{8} & rs174575 & 11 & 61830531 & Colorectal cancer & Enhancer & FADS2 & 61828391 & 2140 & 72 \\
\hline & & & & & & AP002754.2 & 61655702 & 174829 & \\
\hline 9 & rs56069439 & 19 & 17283116 & $\begin{array}{l}\text { Breast cancer and } \\
\text { ovarian cancer }\end{array}$ & Enhancer & ABHD8 & 17301616 & -18500 & 73 \\
\hline \multirow[t]{2}{*}{10} & rs4748812 & 10 & 22550154 & $\begin{array}{l}\text { Childhood acute } \\
\text { lymphoblastic } \\
\text { leukaemia }\end{array}$ & Enhancer & PIP4K2A & 22714326 & -164172 & 74 \\
\hline & rs11591377 & 10 & 22124373 & $\begin{array}{l}\text { Childhood acute } \\
\text { lymphoblastic } \\
\text { leukaemia }\end{array}$ & Enhancer & BMI1 & 22326450 & -202077 & \\
\hline 11 & rs 62510556 & 8 & 117152033 & Type 2 diabetes & Enhancer & SLC30A8 & 116950217 & 201816 & 75 \\
\hline \multirow[t]{10}{*}{12} & $\begin{array}{l}\text { rs7864322 } \\
\text { rs12352658 } \\
\text { rs7847449 and } \\
\text { rs10759944 all in } \\
\text { LD with the GWAS } \\
\text { variant rs965513 }\end{array}$ & 9 & 97786652 & Thyroid cancer & $\begin{array}{l}\text { All variants in the } \\
\text { same enhancer }\end{array}$ & FOXE1 & 97853915 & -67263 & 76 \\
\hline & & & 97789486 & & & FOXE1 & 97853915 & -64429 & \\
\hline & & & 97789616 & & & FOXE1 & 97853915 & -64299 & \\
\hline & & & 97794690 & & & FOXE1 & 97853915 & -59225 & \\
\hline & & & 97793827 & & & FOXE1 & 97853915 & -60088 & \\
\hline & & & 97786652 & & & PTCSC2 & 97853080 & -66428 & \\
\hline & & & 97789486 & & & PTCSC2 & 97853080 & -63594 & \\
\hline & & & 97789616 & & & PTCSC2 & 97853080 & -63464 & \\
\hline & & & 97794690 & & & PTCSC2 & 97853080 & -58390 & \\
\hline & & & 97793827 & & & PTCSC2 & 97853080 & -59253 & \\
\hline 13 & rs1335532 & 1 & 116558335 & $\begin{array}{l}\text { Protection from } \\
\text { multiple sclerosis }\end{array}$ & Enhancer & CD58 & 116570972 & -12637 & 77 \\
\hline 14 & rs2159100 & 12 & 2237227 & Schizophrenia & Enhancer & CACNA1C & 2052987 & 184240 & 78 \\
\hline \multirow[t]{2}{*}{15} & rs3181077 & 3 & 46204161 & Narcolepsy & Enhancer & CCR1 & 46208313 & -4152 & 79 \\
\hline & & & & & & CCR3 & 46163604 & -46163604 & \\
\hline 16 & rs7163757 & 15 & 62099409 & Type 2 diabetes & Enhancer & VPS13C & 61854457 & 244952 & 80 \\
\hline \multirow[t]{2}{*}{17} & rs148314165 & 6 & 137908854 & SLE & Enhancer & TNFAIP3 & 137871228 & 37626 & 81 \\
\hline & rs200820567 & & 137908903 & & & TNFAIP3 & 137871228 & 37675 & \\
\hline \multirow[t]{6}{*}{18} & $\begin{array}{l}\text { rs139767239, } \\
\text { rs115133228, } \\
\text { rs12722502, } \\
\text { rs12722635, in LD } \\
\text { with rs706778 and } \\
\text { rs706779 }\end{array}$ & 10 & 6058827 & T1D & Intronic enhancers & IL2RA & 6062151 & -3324 & 82 \\
\hline & & & 6054123 & & & & 6062151 & -8028 & \\
\hline & & & 6051176 & & & & 6062151 & -10975 & \\
\hline & & & 6051177 & & & & 6062151 & -10974 & \\
\hline & & & 6056986 & & & & 6062151 & -5165 & \\
\hline & & & 6056861 & & & & 6062151 & -5290 & \\
\hline 19 & rs17810546 & 3 & 159947262 & Coeliac disease & Silencer & IL12A & 159989057 & -41795 & 83 \\
\hline
\end{tabular}

bp, base pair; Chr, chromosome; SLE, systemic lupus erythematosus; T1D, type 1 diabetes; TSS, transcription start site.

lncRNAs are a key component of the repertoire of regulatory elements that control cell differentiation and maintain tissue homeostasis. Recently, IncRNAs have been clearly implicated in immune cell lineage commitment and immune responses (table 2). They have also emerged as an important class of molecules implicated in various human cancers as well as cardiovascular, 
Genomic variant

$$
\text { DODOCA ADODODI }
$$

IDODODOGDODODO

Expression of IncRNA

Function of

Function of IncRNA in transcription

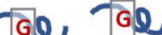

A. Regulation of transcription
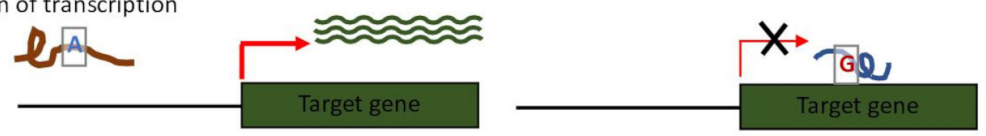

B. Recruitment of components and/or proteins of transcription
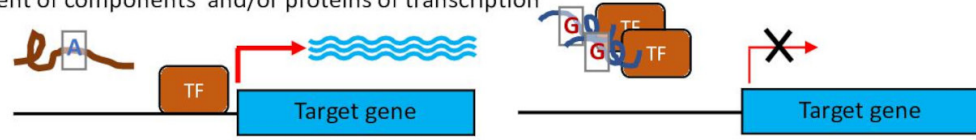

C. Degradation of RNA and splicing modulation
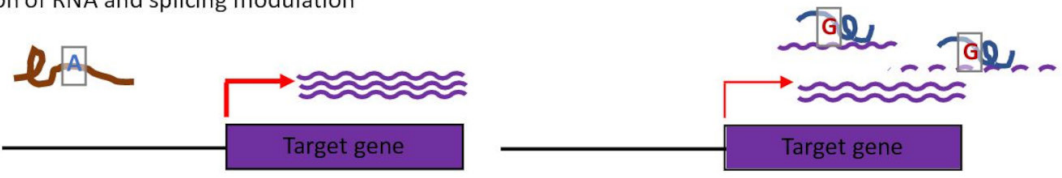

D. Interaction with chromatin remodelling
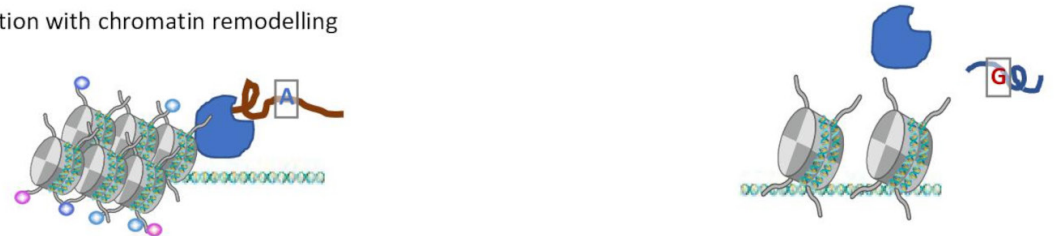

Figure 3 Genetic variants and long non-coding RNAs (IncRNAs). Genetic variants can affect the quantification and function of IncRNAs. (A) IncRQTL, (B) regulation of transcription by IncRNAs, (C) binding of RNAs and IncRNAs, resulting in the formation of alternative splicing isoforms, and (D) interaction of IncRNAs with chromatin-modifying enzymes and nucleosome-remodelling factor. QTL, quantitative trait loci.

neurodegenerative, and autoimmune diseases. ${ }^{43} 44$ If variants with eQTL effects are located on IncRNAs, they may affect transcriptional regulators. ${ }^{20}$ Therefore, the relationship between lncRNAs and risk variants in diseases and traits has received a significant amount of attention and should be the focus of future research.
Genome-wide lncR-eQTL analysis is expected to uncover how GWAS variants relate with lncRNA expression in common diseases and phenotypes. A comprehensive eQTL analysis was previously performed using genome-wide lncRNA expression and genotype data from human peripheral blood mononuclear cells of 43 unrelated individuals. Subsequently, various omics

Table 2 Examples of IncRNAs involved in immune cell functions

\begin{tabular}{|c|c|c|c|}
\hline Cell type & IncRNA & Function & Reference \\
\hline Myeloid cells & Morrbid & Cis regulation of the $\mathrm{Bc}|2| 11$ gene & 84 \\
\hline \multirow[t]{6}{*}{ Monocytes/macrophage } & NTT & Facilitation of cell differentiation and upregulation of IL10 and CXCL10 & 85 \\
\hline & PACER & Supression of Cox2 & 86 \\
\hline & TCON_00019715 & Promotion of macrophages to the $\mathrm{M} 1$ phenotype & 87 \\
\hline & HOTAIR & IkB degradation & 88 \\
\hline & Lnc-MC & Facilitation of monocyte differentiation and upregulation of ACVR1B & 89 \\
\hline & LincRNA-COX2 & $\begin{array}{l}\text { Transcriptional supression of target genes, for example, Ccl5 gene suppression via hnRNPA/B and } \\
\text { hnRNPA2/B1 binding }\end{array}$ & 90 \\
\hline \multirow[t]{4}{*}{ Dendritic cells } & Lnc-DC & Supression of IL6 and IL8 expression via STAT3 binding & 47 \\
\hline & HOTAIRM1 & monocyte/DC differentiation with miR-3960 & 91 \\
\hline & NEAT1 & Induction of tolerogenic phenotype in DC & 92 \\
\hline & Malat1 & Induction of tolerogenic phenotype in DC & 9394 \\
\hline \multirow[t]{7}{*}{ T cell } & NeST & Upregulation IFNG gene expression & 95 \\
\hline & NRON & Regulation of NFAT & 96 \\
\hline & TH2-LCR & Regulation of cytokine expression in Th2 cells & 97 \\
\hline & LncRNA-CD244 & Inhibition of IFNG and TNF & 98 \\
\hline & Linc-MAF-4 & Regulation of MAF expression & 42 \\
\hline & LicR-Ccr2-5'AS & Upreguration of Ccr gene expression & 99 \\
\hline & NKILA & Inhibition of NF-kB activity & 100 \\
\hline
\end{tabular}


integrative network analyses were applied to construct variantlncRNA-mRNA interaction networks, resulting in the detection of 707 pairs of cis-effect associations $(p<5.64 \mathrm{E}-06)$ and 6657 trans-effect associations $(\mathrm{p}<3.51 \mathrm{E}-08) .{ }^{45}$

The integration of genetic data sets from 1829 expression profiles in the FANTOM5 project uncovered the existence of nearly 20000 functional lncRNAs. Approximately 2000 lncRNAs were expressed in significantly associated tissue/ trait pairs. For example, the association between the middle temporal gyrus and autism spectrum disorder involves 18 lncRNAs from 49 genes. ${ }^{20}$ On the other hand, a genetic variant over-represented in patients with coeliac disease lies within $\operatorname{lnc}$ 13 , reduces its binding affinity for HDAC1, and weakens the epigenetic repression of inflammatory genes. ${ }^{46}$ lncRNAs regulate multiple processes in disparate subsets of immune cells, such as the intensity of STAT3 signalling in human dendritic cells, ${ }^{47}$ Th1 versus Th2 differentiation of human lymphocytes, ${ }^{42}$ and perhaps not surprisingly, the epigenetics-mediated training of immune responses in human monocytes. ${ }^{41}$ Thus, IncRNAs play an important role in gene regulation, and their profiling can reveal associations with specific tissues and cells.

\section{FURTHER ANALYSES FOR FUNCTIONAL GENOMICS}

Several different analyses have been used to annotate putative causal variants in GWASs, such as risk-associated SNPs in the promoter and enhancer regions (figure $2 \mathrm{~B}$; table 1 ). ${ }^{48}$ Some of the functions of risk-associated enhancers and promoter variants have been experimentally proven, while others have been inferred from positional information; however, the functions of enhancers are particularly difficult to prove. In fact, the functions of many risk variants that exist outside of gene bodies remain unclear. Enhancers may be far away from the TSS, even further than $1 \mathrm{Mb}$. Transcriptional regulation from distal locations is facilitated by the folding of the genome, which places regulatory elements in three-dimensional proximity of the TSS. Advances in analytical techniques and next-generation sequencing techniques, like $\mathrm{Hi}-\mathrm{C}$-sequencing and micro-C-sequencing, allow the identification of trans-acting regulatory elements as well as cis -acting elements ${ }^{4-51}$ (figure $2 \mathrm{~B}$ ). Integrated analysis of omics data is also currently undergoing various statistical advances.

Biological analysis of regions regulated by TFs that were previously analysed by reporter assays can now be comprehensively performed through massive parallel reporter assays (MPRAs). In MPRAs, a library of barcoded candidate regulatory sequences is generated, cloned in the backbone of a reporter plasmid, and then transfected into an appropriate cell line. RNA-seq then allows the precise identification and quantitation of the transcriptional output associated with each regulatory variant. MPRAs facilitates the high-resolution, quantitative dissection of sequence-activity relationships of transcriptional regulatory elements with high reproducibility. MPRAs were developed to test the function of enhancer sequences in combination with targeted mutagenesis ${ }^{52} 53$ and have subsequently been exploited to test human promoters, enhancers ${ }^{54}$ and eQTL. ${ }^{55}$ However, episomal reporter plasmids do not allow the testing of candidate regulatory sequences in their original context, a limitation that may affect the validation of epigenetic QTL. Chromatinised adenoviral and lentiviral vectors have therefore been developed to circumvent this limitation. ${ }^{5758}$

A new type of screening assay based on CRISPR/Cas9 technology examines the functions of regulatory regions in their original genomic context. CRISPR/Cas9 is used to test the function of candidate regulatory regions by targeted genomic deletion. ${ }^{59}$ Other
CRISPR-based screening approaches, such as CRISPR/Cas9-mediated activation and inactivation, have been coupled with RNA-seqbased transcriptional quantification. However, these methods have not yet been scaled to the genome-wide level. ${ }^{60}$ Furthermore, new approaches that combine CRISPR-based genome engineering with patient-specific induced pluripotent stem cell-based models are powerful tools for identifying putative causal loci that regulate gene expression and cellular functions. ${ }^{61}$

Advances in next-generation sequencing have improved single-cell gene expression analysis. Bulk RNA-seq experiments, commonly used in transcriptomic studies, examine the average gene expression among sampled cells and thus mask cell heterogeneity. However, single-cell RNA sequencing (scRNA-seq) can be used to reveal the heterogeneity of gene expression between various cells. Largescale scRNA-seq can reveal patterns of gene, isoform, and allelic expression across different cell types and cell conditions. ${ }^{62}$ Most scRNA-seq methods use unique molecular identifiers (UMIs) of specific allelic origin. ${ }^{62}$ Utilisation of UMIs confers an important advantage to eQTL analysis, since they are useful to precisely understand allele imbalance. However, scRNA-seq analysis methods have low detection sensitivity and may not be able to detect many genes with low levels of expression, suggesting a need for improvement. ${ }^{63}$ New statistical analyses have been developed to enable cell type identification, such as deconvolution analysis, which uses scRNA-seq and reference panel data. In addition to the transcriptomic analysis, scRNA-seq is also used in epigenomic analyses (eg, single-cell ChIPsequencing). Additionally, spatial transcriptomics methods are also improving markedly. ${ }^{64}$ In the future, spatial transcriptomics methods may enable the visualisation, while retaining the spatial information of the tissue.

\section{CONCLUSIONS}

As described above, considerable progress has been made in understanding the genetics and pathogenesis of autoimmune diseases. However, our understanding of the causality of these diseases is inadequate, and the development of new therapies based on genomic information for these diseases is required. Therefore, we need to apply a variety of approaches to identify the missing links.

GWASs are based on the hypothesis that commonly occurring diseases involve commonly occurring variants, with a large number of variants being involved in the development of a disease. Accordingly, functional analyses of risk variants are complicated. Statistical associations between genes and diseases alone cannot identify the risk variants that contribute to pathogenesis. Integration of GWAS data and omics data has the potential to elucidate the function of each risk variant while also identifying disease and tissue specificity. These findings will help reveal a more detailed mechanism of pathogenesis of autoimmune diseases and thus lead to their precise diagnosis and treatment.

Contributors AS and MMG carried out the experiment. AS wrote the manuscript with support from $\mathrm{KY}$ and MMG. KY supervised the project.

Funding This study was funded by Japan Society for the Promotion of Science (grant number 18H05285) and Chugai Pharmaceutical Co.

Competing interests KY has received grants from Chugai Pharmaceutical $\mathrm{Co}$.

Patient and public involvement Patients and/or the public were not involved in the design, or conduct, or reporting, or dissemination plans of this research.

Patient consent for publication Not required.

Ethics approval Our study is performed in accordance with the International Conference on Harmonisation Guidelines for Good Clinical Practice and the Declaration of Helsinki, and the study protocols were approved by ethics review boards of RIKEN. 
Provenance and peer review Not commissioned; externally peer reviewed.

\section{ORCID iD}

Kazuhiko Yamamoto http://orcid.org/0000-0001-9037-3625

\section{REFERENCES}

1 Gallagher MD, Chen-Plotkin AS. The Post-GWAS era: from association to function. Am J Hum Genet 2018:102:717-30.

2 Welter D, MacArthur J, Morales J, et al. The NHGRI GWAS catalog, a curated resource of SNP-trait associations. Nucleic Acids Res 2014:42:D1001-6.

3 Farh KK-H, Marson A, Zhu J, et al. Genetic and epigenetic fine mapping of causal autoimmune disease variants. Nature 2015;518:337-43.

4 Gabriel SB, Schaffner SF, Nguyen H, et al. The structure of haplotype blocks in the human genome. Science 2002;296:2225-9.

5 Edwards SL, Beesley J, French JD, et al. Beyond GWASs: illuminating the dark road from association to function. Am J Hum Genet 2013;93:779-97.

6 Maurano MT, Humbert R, Rynes E, et al. Systematic localization of common diseaseassociated variation in regulatory DNA. Science 2012;337:1190-5.

7 Ishigaki K, Kochi Y, Yamamoto K. Genetics of human autoimmunity: from genetic information to functional insights. Clin Immunol 2018;186:9-13.

8 Garrido-Cardenas JA, Mesa-Valle C, Manzano-Agugliaro F. Trends in plant research using molecular markers. Planta 2018;247:543-57.

9 Ye Y, Zhang Z, Liu Y, et al. A multi-omics perspective of quantitative trait loci in precision medicine. Trends Genet 2020;36:318-36.

10 Akiyama M, Ishigaki K, Sakaue S, et al. Characterizing rare and low-frequency height-associated variants in the Japanese population. Nat Commun 2019;10:4393

11 Kowalski MH, Oian H, Hou Z, et al. Use of $>100,000$ NHLBI Trans-Omics for Precision Medicine (TOPMed) Consortium whole genome sequences improves imputation quality and detection of rare variant associations in admixed African and Hispanic/Latino populations. PLoS Genet 2019;15:e1008500.

12 Gerrits A, Li Y, Tesson BM, et al. Expression quantitative trait loci are highly sensitive to cellular differentiation state. PLoS Genet 2009:5:e1000692.

13 Albert FW, Kruglyak $L$. The role of regulatory variation in complex traits and disease. Nat Rev Genet 2015;16:197-212.

14 Gibson G, Powell JE, Marigorta UM. Expression quantitative trait locus analysis for translational medicine. Genome Med 2015:7:60.

15 Patel L, Kang R, Rosenberg SC, et al. Dynamic reorganization of the genome shapes the recombination landscape in meiotic prophase. Nat Struct Mol Biol 2019;26:164-74

16 Braunschweig U, Gueroussov S, Plocik AM, et al. Dynamic integration of splicing within gene regulatory pathways. Cell 2013;152:1252-69.

17 Chen L, Ge B, Casale FP, et al. Genetic drivers of epigenetic and transcriptional variation in human immune cells. Cell 2016;167:e24:1398-414.

18 Garieri M, Delaneau 0, Santoni F, et al. The effect of genetic variation on promoter usage and enhancer activity. Nat Commun 2017:8:1358.

19 Zhao Q, Dacre M, Nguyen T, et al. Molecular mechanisms of coronary disease revealed using quantitative trait loci for TCF21 binding, chromatin accessibility, and chromosomal looping. Genome Biol 2020;21:135

20 Hon C-C, Ramilowski JA, Harshbarger J, et al. An atlas of human long non-coding RNAs with accurate 5' ends. Nature 2017:543:199-204.

21 Budach S, Heinig M, Marsico A. Principles of microRNA regulation revealed through modeling microRNA expression quantitative trait loci. Genetics 2016;203:1629-40.

22 Haberle V, Stark A. Eukaryotic core promoters and the functional basis of transcription initiation. Nat Rev Mol Cell Biol 2018;19:621-37.

23 Hampsey M. Molecular genetics of the RNA polymerase II general transcriptional machinery. Microbiol Mol Biol Rev 1998;62:465-503.

24 Banerji J, Rusconi S, Schaffner W. Expression of a beta-globin gene is enhanced by remote SV40 DNA sequences. Cell 1981:27:299-308.

25 Shlyueva D, Stampfel G, Stark A. Transcriptional enhancers: from properties to genome-wide predictions. Nat Rev Genet 2014;15:272-86.

26 Spitz F, Furlong EEM. Transcription factors: from enhancer binding to developmental control. Nat Rev Genet 2012;13:613-26.

27 Kumar A, Bansal M. Unveiling DNA structural features of promoters associated with various types of TSSs in prokaryotic transcriptomes and their role in gene expression. DNA Res 2017:24:25-35.

28 Wu J, Metz C, Xu X, et al. A novel polymorphic CAAT/enhancer-binding protein beta element in the FasL gene promoter alters Fas ligand expression: a candidate background gene in African American systemic lupus erythematosus patients. J Immunol 2003:170:132-8.

29 Kochi Y, Yamada R, Suzuki A, et al. A functional variant in FCRL3, encoding Fc receptor-like 3 , is associated with rheumatoid arthritis and several autoimmunities. Nat Genet 2005;37:478-85.

30 Hirabayashi S, Bhagat S, Matsuki Y, et al. NET-CAGE characterizes the dynamics and topology of human transcribed cis-regulatory elements. Nat Genet 2019;51:1369-79.
31 Clyde DE, Corado MSG, Wu X, et al. A self-organizing system of repressor gradients establishes segmental complexity in Drosophila. Nature 2003:426:849-53.

32 Kaikkonen MU, Spann NJ, Heinz S, et al. Remodeling of the enhancer landscape during macrophage activation is coupled to enhancer transcription. Mol Cell 2013:51:310-25.

33 Andersson R, Sandelin A. Determinants of enhancer and promoter activities of regulatory elements. Nat Rev Genet 2020;21:71-87.

34 Ernst J, Kheradpour P, Mikkelsen TS, et al. Mapping and analysis of chromatin state dynamics in nine human cell types. Nature 2011;473:43-9.

35 Corradin 0, Scacheri PC. Enhancer variants: evaluating functions in common disease. Genome Med 2014:6:85.

36 Woolfe A, Goodson M, Goode DK, et al. Highly conserved non-coding sequences are associated with vertebrate development. PLOS Biol 2005:3:e7.

37 Yáñez-Cuna JO, Kvon EZ, Stark A. Deciphering the transcriptional cis-regulatory code. Trends Genet 2013;29:11-22.

38 Jiang C, Li Y, Zhao Z, et al. Identifying and functionally characterizing tissue-specific and ubiquitously expressed human IncRNAs. Oncotarget 2016;7:7120-33.

39 Shiraki T, Kondo S, Katayama S, et al. Cap analysis gene expression for highthroughput analysis of transcriptional starting point and identification of promoter usage. Proc Natl Acad Sci U SA 2003;100:15776-81.

40 De Santa F, Barozzi I, Mietton F, et al. A large fraction of extragenic RNA Pol II transcription sites overlap enhancers. PLoS Biol 2010:8:e1000384.

41 Fanucchi S, Fok ET, Dalla E, et al. Immune genes are primed for robust transcription by proximal long noncoding RNAs located in nuclear compartments. Nat Genet 2019;51:138-50.

42 Ranzani V, Rossetti G, Panzeri I, et al. The long intergenic noncoding RNA landscape of human lymphocytes highlights the regulation of T cell differentiation by lincMAF-4. Nat Immunol 2015;16:318-25.

43 Esteller M. Non-Coding RNAs in human disease. Nat Rev Genet 2011;12:861-74

44 Han $\mathrm{P}$, Chang C-P. Long non-coding RNA and chromatin remodeling. RNA Biol 2015:12:1094-8

45 Xia W, Zhu X-W, Mo X-B, et al. Integrative multi-omics analysis revealed SNPIncRNA-mRNA (SLM) networks in human peripheral blood mononuclear cells. Hum Genet 2017;136:451-62

46 Castellanos-Rubio A, Fernandez-Jimenez N, Kratchmarov R, et al. A long noncoding RNA associated with susceptibility to celiac disease. Science 2016;352:91-5.

47 Wang P, Xue Y, Han Y, et al. The STAT3-binding long noncoding RNA Inc-DC controls human dendritic cell differentiation. Science 2014;344:310-3

48 Ishigaki K, Kochi Y, Suzuki A, et al. Polygenic burdens on cell-specific pathways underlie the risk of rheumatoid arthritis. Nat Genet 2017;49:1120-5.

49 Stevens TJ, Lando D, Basu S, et al. 3D structures of individual mammalian genomes studied by single-cell Hi-C. Nature 2017;544:59-64.

50 de Souza N, Genomics deSN. Genomics. Micro-C maps of genome structure. Nat Methods 2015:12:812.

51 Lu L, Liu X, Huang W-K, et al. Robust Hi-C maps of enhancer-promoter interactions reveal the function of non-coding genome in neural development and diseases. $\mathrm{Mol}$ Cell 2020;79:e15:521-34.

52 Melnikov A, Murugan A, Zhang $X$, et al. Systematic dissection and optimization of inducible enhancers in human cells using a massively parallel reporter assay. Nat Biotechnol 2012:30:271-7.

53 Smith RP, Taher L, Patwardhan RP, et al. Massively parallel decoding of mammalian regulatory sequences supports a flexible organizational model. Nat Genet 2013:45:1021-8

54 Nguyen TA, Jones RD, Snavely AR, et al. High-Throughput functional comparison of promoter and enhancer activities. Genome Res 2016:26:1023-33.

55 Tewhey R, Kotliar D, Park DS, et al. Direct identification of hundreds of ExpressionModulating variants using a multiplexed reporter assay. Cell 2016:165:1519-29.

56 Ulirsch JC, Nandakumar SK, Wang L, et al. Systematic functional dissection of common genetic variation affecting red blood cell traits. Cell 2016:165:1530-45.

57 Inoue F, Kircher M, Martin B, et al. A systematic comparison reveals substantial differences in chromosomal versus episomal encoding of enhancer activity. Genome Res 2017:27:38-52.

58 Shen SQ, Myers CA, Hughes AEO, et al. Massively parallel cis-regulatory analysis in the mammalian central nervous system. Genome Res 2016:26:238-55.

59 Rajagopal N, Srinivasan S, Kooshesh K, et al. High-Throughput mapping of regulatory DNA. Nat Biotechnol 2016:34:167-74.

60 Xie S, Duan J, Li B, et al. Multiplexed engineering and analysis of combinatorial enhancer activity in single cells. Mol Cell 2017;66:285-99.

61 Bassett AR. Editing the genome of hiPSC with CRISPR/Cas9: disease models. Mamm Genome 2017:28:348-64.

62 Hagemann-Jensen M, Ziegenhain C, Chen P, et al. Single-Cell RNA counting at allele and isoform resolution using Smart-seq3. Nat Biotechnol 2020;38:708-14.

63 Bagnoli JW, Ziegenhain C, Janjic A, et al. Sensitive and powerful single-cell RNA sequencing using mcSCRB-seq. Nat Commun 2018;9:2937.

64 Burgess DJ. Spatial transcriptomics coming of age. Nat Rev Genet 2019;20:317.

65 Allen EK, Randolph AG, Bhangale T, et al. SNP-mediated disruption of CTCF binding at the IFITM3 promoter is associated with risk of severe influenza in humans. Nat Med 2017;23:975-83. 
66 Gutierrez-Arcelus M, Baglaenko Y, Arora J, et al. Allele-Specific expression changes dynamically during T cell activation in HLA and other autoimmune loci. Nat Genet 2020;52:247-53.

67 Putlyaeva LV, Demin DE, Korneev KV, et al. Potential Markers of Autoimmune Diseases, Alleles rs115662534(T) and rs548231435(C), Disrupt the Binding of Transcription Factors STAT1 and EBF1 to the Regulatory Elements of Human CD40 Gene. Biochemistry 2018;83:1534-42.

68 Sun W, Wu H-Y, Chen S. Influence of TBX21 T-1993C variant on autoimmune hepatitis development by yin-yang 1 binding. World J Gastroenterol 2017;23:8500-11.

69 Wen L, Zhu C, Zhu Z, et al. Exome-wide association study identifies four novel loci for systemic lupus erythematosus in Han Chinese population. Ann Rheum Dis 2018;77:417.

70 Deng Y, Zhao J, Sakurai D, et al. Decreased SMG7 expression associates with lupus-risk variants and elevated antinuclear antibody production. Ann Rheum Dis 2016;75:2007-13.

71 Chen X-F, Zhu D-L, Yang M, et al. An osteoporosis risk SNP at 1 p36.12 acts as an allele-specific enhancer to modulate LINC00339 expression via long-range loop formation. Am J Hum Genet 2018;102:776-93.

72 Tian J, Lou J, Cai Y, et al. Risk SNP-Mediated Enhancer-Promoter Interaction Drives Colorectal Cancer through Both FADS2 and AP002754.2. Cancer Res 2020;80:1804-18.

73 Lawrenson K, Kar S, McCue K, et al. Functional mechanisms underlying pleiotropic risk alleles at the 19p13.1 breast-ovarian cancer susceptibility locus. Nat Commun 2016;7:12675.

74 de Smith AJ, Walsh KM, Francis SS, et al. Bmi1 enhancer polymorphism underlies chromosome 10p12.31 association with childhood acute lymphoblastic leukemia. Int J Cancer 2018;143:2647-58.

75 Pound LD, Sarkar SA, Cauchi S, et al. Characterization of the human SIc30a8 promoter and intronic enhancer. J Mol Endocrinol 2011;47:251-9.

$76 \mathrm{He} \mathrm{H}$, Li W, Liyanarachchi S, et al. Multiple functional variants in long-range enhancer elements contribute to the risk of SNP rs965513 in thyroid cancer. Proc Natl Acad Sci USA 2015;112:6128-33.

77 Mitkin NA, Muratova AM, Korneev KV, et al. Protective C allele of the singlenucleotide polymorphism rs 1335532 is associated with strong binding of ASCL2 transcription factor and elevated CD58 expression in B-cells. Biochim Biophys Acta Mol Basis Dis 2018;1864:3211-20.

78 Roussos P, Mitchell AC, Voloudakis G, et al. A role for noncoding variation in schizophrenia. Cell Rep 2014;9:1417-29.

79 Toyoda H, Miyagawa T, Koike A, et al. A polymorphism in CCR1/CCR3 is associated with narcolepsy. Brain Behav Immun 2015;49:148-55.

80 Mehta ZB, Fine N, Pullen TJ, et al. Changes in the expression of the type 2 diabetesassociated gene VPS13C in the $\beta$-cell are associated with glucose intolerance in humans and mice. Am J Physiol Endocrinol Metab 2016;311:E488-507.

81 Wang S, Wen F, Tessneer KL, et al. Talen-Mediated enhancer knockout influences TNFAIP3 gene expression and mimics a molecular phenotype associated with systemic lupus erythematosus. Genes Immun 2016;17:165-70.

82 Schwartz AM, Demin DE, Vorontsov IE, et al. Multiple single nucleotide polymorphisms in the first intron of the IL2RA gene affect transcription factor binding and enhancer activity. Gene 2017;602:50-6.
83 Zwiers A, van Wanrooij RLJ, Dieckman T, et al. Celiac disease associated SNP rs17810546 is located in a gene silencing region. Gene 2020;726:144165.

84 Mowel WK, Kotzin JJ, McCright SJ, et al. Control of immune cell homeostasis and function by IncRNAs. Trends Immunol 2018:39:55-69.

85 Yang C-A, Li J-P, Yen J-C, et al. IncRNA NTT/PBOV1 axis promotes monocyte differentiation and is elevated in rheumatoid arthritis. Int J Mol Sci 2018;19. doi:10.3390/ijms19092806. [Epub ahead of print: 18 Sep 2018].

86 Krawczyk M, Emerson BM. p50-associated COX-2 extragenic RNA (PACER) activates COX-2 gene expression by occluding repressive NF- $\kappa B$ complexes. Elife 2014;3:e01776.

87 Xie Y, Wang M, Tian J, et al. Long non-coding RNA expressed in macrophage co-varies with the inflammatory phenotype during macrophage development and polarization. J Cell Mol Med 2019;23:6530-42.

88 Meredith EK, Balas MM, Sindy K, et al. An RNA matchmaker protein regulates the activity of the long noncoding RNA HOTAIR. RNA 2016;22:995-1010.

89 Chen M-T, Lin H-S, Shen C, et al. PU.1-Regulated long noncoding RNA Inc-MC controls human monocyte/macrophage differentiation through interaction with microRNA 199a-5p. Mol Cell Biol 2015;35:MCB.00429-15-24.

90 Basavappa M, Cherry S, Henao-Mejia J. Long noncoding RNAs and the regulation of innate immunity and host-virus interactions. J Leukoc Biol 2019;106:83-93.

91 Xin J, Li J, Feng Y, et al. Downregulation of long noncoding RNA HOTAIRM1 promotes monocyte/dendritic cell differentiation through competitively binding to endogenous miR-3960. Onco Targets Ther 2017;10:1307-15.

92 Shui $X$, Chen S, Lin J, et al. Knockdown of IncRNA NEAT1 inhibits Th17/CD4 ${ }^{+}$ T cell differentiation through reducing the STAT3 protein level. J Cell Physiol 2019;234:22477-84.

93 Zhang M, Zheng Y, Sun Y, et al. Knockdown of NEAT1 induces tolerogenic phenotype in dendritic cells by inhibiting activation of NLRP3 inflammasome. Theranostics 2019;9:3425-42.

94 Zhang Y, Zhang G, Liu Y, et al. GDF15 regulates MALAT-1 circular RNA and inactivates NFKB signaling leading to immune tolerogenic DCs fo preventing alloimmune rejection in heart transplantation. Front Immunol 2018;9:2407.

95 Gomez JA, Wapinski OL, Yang YW, et al. The nest long ncRNA controls microbial susceptibility and epigenetic activation of the interferon- $\gamma$ locus. Cell 2013;152:743-54

96 Imam H, Bano AS, Patel P, et al. The IncRNA NRON modulates HIV-1 replication in a NFAT-dependent manner and is differentially regulated by early and late viral proteins. Sci Rep 2015:5:8639.

97 Spurlock CF. 3Rd, Tossberg JT, Guo Y, collier sp, Crooke PS, 3rd, Aune TM. expression and functions of long noncoding RNAs during human T helper cell differentiation. Nat Commun 2015:6:6932.

98 Wang $\mathrm{Y}$, Zhong $\mathrm{H}$, Xie $\mathrm{X}$, et al. Long noncoding RNA derived from CD244 signaling epigenetically controls CD8+T-cell immune responses in tuberculosis infection. Proc Natl Acad Sci U S A 2015;112:E3883-92.

99 Chen J, Ao L, Yang J. Long non-coding RNAs in diseases related to inflammation and immunity. Ann Trans/ Med 2019;7:494.

100 Zhang W, Guo Q, Liu G, et al. NKILA represses nasopharyngeal carcinoma carcinogenesis and metastasis by NF- $\mathrm{KB}$ pathway inhibition. PLoS Genet 2019;15:e1008325. 